\title{
Immediate Cardiovascular Effects of a Single Yoga Session in Different Conditions
}

\author{
Ananda Balayogi Bhavanani ${ }^{1 *}$, Meena Ramanathan ${ }^{1}$ and Madanmohan ${ }^{2}$ \\ ${ }^{1}$ Centre for Yoga Therapy, Education and Research, Mahatma Gandhi Medical College \& Research Institute, Pillayarkuppam, Pondicherry, India \\ ${ }^{2}$ Department of Physiology, Mahatma Gandhi Medical College \& Research Institute, Pondicherry, India
}

\begin{abstract}
Aim and objective: This retrospective review of clinical data was done to determine cardiovascular effects of a single yoga session in normal subjects as well as patients of different medical conditions.

Methods: Data of 1896 patients (1229 female, 633 male and 34 transgender) with mean age of $36.28 \pm 12.64$ y who attended yoga therapy sessions at CYTER between November 2010 and September 2012 was used for analysis. Heart rate (HR), systolic (SP) and diastolic pressure (DP) had been recorded using non-invasive blood pressure (NIBP) apparatus before and after 60 minute yoga sessions at CYTER and indices like pulse pressure (PP), mean pressure (MP), rate-pressure product (RPP) and double product (DoP) were derived from recorded parameters. Participants were undergoing appropriate yoga therapy protocols as per their individual condition while normal subjects had a general schedule of practice. Typical yoga sessions included simple warm ups (jathis and surya namaskar), breath body movement coordination practices (kriyas), static stretching postures (asana), breathing techniques (pranayama), relaxation and chanting.

Results: There were statistically significant $(p<0.001)$ reductions in all the studied cardiovascular parameters following the yoga session. The magnitude of reductions differed in the groups, it being more significant in those having hypertension $(n=505)$ and less significant in those having endocrine/skin $(n=230)$ and musculoskeletal $(n=120)$ conditions. It was moderately significant in the normal subjects $(n=582)$ as well as patients having psychiatric $(n=302)$ and respiratory $(n=157)$ conditions.

Conclusion: There is a healthy reduction in HR, BP and derived cardiovascular indices following a single yoga session. The magnitude of this reduction depends on the pre-existing medical condition as well as the yoga therapy protocol adopted. These changes may be attributed to enhanced harmony of cardiac autonomic function as a result of coordinated breath-body work and mind-body relaxation due to yoga.
\end{abstract}

Keywords: Yoga therapy; Cardiovascular effects; Psycho-somatic harmony

\section{Introduction}

Humanity is today faced with numerous debilitating chronic illnesses related to aging, environment and an increasingly hedonistic lifestyle. These illnesses include cancer, diabetes, osteoporosis, and cardiovascular disease, as well as incurable diseases such as AIDS. While modern medicine has much to offer in its treatment of acute illness, accidents and communicable diseases, it cannot provide all the solutions for the many ills that plague $21^{\text {st }}$ century (woman). Yoga, as a complement to modern medicine, can be especially useful in helping to fill in the gaps in the fields of disease prevention, management and rehabilitation. When combined, modern medicine and yoga turn out to be more than the sum of their parts. What is the source of this synergy between modern and ancient science? While modern science looks outward for the cause of all ills, the yogi searches the depths of their own self, finding therein many of the answers he needs to maintain a vital equilibrium. The combination of the outward and inward search proves to be more effective than either alone.

Dr. Dean Ornish, the renowned American physician and author who has shown that a yogic lifestyle can reverse heart disease, says, "Yoga is a system of perfect tools for achieving union as well as healing" [1]. Dr. B. Ramamurthy, the eminent neurosurgeon, has observed that yoga practice reorients the functional hierarchy of the entire nervous system [1]. He has also noted that yoga benefits the cardiovascular, respiratory, digestive, and endocrine systems, in addition to bringing about other positive biochemical changes. For humanity to take full advantage of its birthright of health and happiness, it is imperative that modern, science-based medicine integrate the holistic approach of traditional healing techniques like yoga. Only in this way can medical practitioners provide true health care, as opposed to merely caring for the sick. The result will be an improvement in the quality of health, and life, around the world.

Numerous studies have been done in the past few decades on psycho-physiological and biochemical changes occurring following practice of yoga [2-9]. A few clinical trials have also shown promise despite yoga not being ideally suited for the scientific gold standard of 'double-blind' clinical trials $[10,11]$ and though we are truly yet to research and understand subtler effects of yoga [12]. Evidence is also growing that yoga practice is a relatively low-risk, high-yield approach to improving overall health and wellbeing [13]. It has been rightly pointed out that yoga is qualitatively different from any other mode of physical activity in that it consists of a unique combination of isometric

*Corresponding author: Ananda Balayogi Bhavanani, CYTER, Mahatma Gandh Medical College \& Research Institute, Pillayarkuppam, Pondicherry 607402, India, Tel: 91-413-2622902; E-mail: yognat@gmail.com

Received September 28, 2013; Accepted November 11, 2013; Published November 13, 2013

Citation: Bhavanani AB, Ramanathan M, Madanmohan (2013) Immediate Cardiovascular Effects of a Single Yoga Session in Different Conditions. Altern Integ Med 2: 144. doi:10.4172/2327-5162.1000144

Copyright: (C) 2013 Bhavanani AB, et al. This is an open-access article distributed under the terms of the Creative Commons Attribution License, which permits unrestricted use, distribution, and reproduction in any medium, provided the original author and source are credited. 
Citation: Bhavanani AB, Ramanathan M, Madanmohan (2013) Immediate Cardiovascular Effects of a Single Yoga Session in Different Conditions. Altern Integ Med 2: 144. doi:10.4172/2327-5162.1000144

Page 2 of 4

muscular contractions, stretching exercises, relaxation techniques, and breathing exercises [14].

There are only a few studies that have focused on the immediate effects of a single yoga session and these include one that investigated the effectiveness of a single 90-minute hatha yoga class and concluded that it can significantly reduce perceived stress [15]. Another recent study showed that cognitive performance after a yoga session was significantly superior as compared with an aerobic session [16]. A recent report on the acute effects of one session of hatha yoga practice on blood pressure and other cardiovascular responses in healthy volunteers has showed that systolic (SP), mean (MP), and diastolic (DP) blood pressures increased significantly during the yoga practice [14]. However they have not compared the pre-post effects of the entire session and only focused on the individual techniques during the session.

With the above in mind, this retrospective review of data was done to determine cardiovascular effects of a single 60 -minute yoga session in normal subjects as well as patients of different medical conditions.

\section{Materials and Methods}

This retrospective review of clinical data was conducted at the Center for Yoga Therapy Education and Research (CYTER) functioning in Mahatma Gandhi Medical College and Research Institute, Puducherry,
India. CYTER is conducting regular yoga therapy sessions daily for patients of different conditions as well as normal subjects. Data of 1896 patients ( 1229 female, 633 male and 34 transgender) with mean age of $36.28 \pm 12.64 \mathrm{y}$ who attended yoga therapy sessions at CYTER between November 2010 and September 2012 was used for analysis. These sessions were carried out in CYTER Yoga hall between $10 \mathrm{AM}$ and 12 noon on weekdays in a quiet environment, with a comfortable temperature and subdued lighting. The participants had been advised to finish their breakfast at least 2 hours earlier and come after emptying bowel and bladder.

Heart rate (HR), SP and DP had been recorded before and after the 60 minute yoga sessions at CYTER. To ensure objectivity, all recordings were performed using non-invasive semi-automatic BP monitor $(\mathrm{CH}-$ 432, Citizen Systems, Tokyo, Japan) with an instrumental accuracy of $\pm 5 \%$ for HR and $\pm 3 \mathrm{~mm} \mathrm{Hg}$ for BP. Pre intervention HR and BP was recorded after $5 \mathrm{~min}$ of supine rest while the post-intervention recordings were taken at the end of the session in supine position. Cardiovascular indices like pulse pressure (PP), MP, rate-pressure product (RPP) and double product (DoP) were derived from the recorded parameters.

The participants were undergoing appropriate yoga therapy protocols as per their individual condition (Table 1) while normal

\begin{tabular}{|c|c|c|c|c|c|c|}
\hline Yogic technique & $\begin{array}{l}\text { Normal } \\
\text { subjects }\end{array}$ & $\begin{array}{l}\text { Endocrine and } \\
\text { skin disorders }\end{array}$ & $\begin{array}{l}\text { Respiratory } \\
\text { disorders }\end{array}$ & $\begin{array}{l}\text { Hypertension \& } \\
\text { CV disorders }\end{array}$ & $\begin{array}{l}\text { Musculo-skeletal } \\
\text { disorders }\end{array}$ & $\begin{array}{l}\text { Psychiatric } \\
\text { disorders }\end{array}$ \\
\hline Jathis \& kriyas (loosening techniques) & $\sqrt{ }$ & $\sqrt{ }$ & $\sqrt{ }$ & $\sqrt{ }$ & $\sqrt{ }$ & $\sqrt{ }$ \\
\hline Aruna surya namaskar (slow, breath coordinated) & $\sqrt{ }$ & $\sqrt{ }$ & & & & $\sqrt{ }$ \\
\hline \multicolumn{7}{|l|}{ Standing asanas } \\
\hline Trikona asana & $\sqrt{ }$ & $\sqrt{ }$ & $\sqrt{ }$ & & $\sqrt{ }$ & \\
\hline Veera asana $1 \& 2$ & $\sqrt{ }$ & & $\sqrt{ }$ & & & $\sqrt{ }$ \\
\hline Tada asana & & $\sqrt{ }$ & & $\sqrt{ }$ & $\sqrt{ }$ & $\sqrt{ }$ \\
\hline Ardhakati \& kati chakra asana & & $\sqrt{ }$ & $\sqrt{ }$ & & $\sqrt{ }$ & \\
\hline Mehru asana \& nasarga mukha bhastrika & & & & $\sqrt{ }$ & & $\sqrt{ }$ \\
\hline Ardha utkat asana & & & & & $\sqrt{ }$ & $\sqrt{ }$ \\
\hline \multicolumn{7}{|l|}{ Sitting asanas } \\
\hline Vakra asana & $\sqrt{ }$ & $\sqrt{ }$ & $\sqrt{ }$ & $\sqrt{ }$ & $\sqrt{ }$ & \\
\hline Paschimottana/purvottana asana & $\sqrt{ }$ & $\sqrt{ }$ & $\sqrt{ }$ & & $\sqrt{ }$ & $\sqrt{ }$ \\
\hline Matsya asana & $\sqrt{ }$ & & $\sqrt{ }$ & $\sqrt{ }$ & & $\sqrt{ }$ \\
\hline Chatuspada kriya/vyagraha pranayama & $\sqrt{ }$ & & & $\sqrt{ }$ & $\sqrt{ }$ & \\
\hline Danda kriya & $\sqrt{ }$ & & & & $\sqrt{ }$ & \\
\hline Ashwini mudra/moola bandha & & & & & & $\sqrt{ }$ \\
\hline \multicolumn{7}{|l|}{ Face prone asanas } \\
\hline Bhujanga asana/ bhujangini mudra & $\sqrt{ }$ & $\sqrt{ }$ & $\sqrt{ }$ & & $\sqrt{ }$ & $\sqrt{ }$ \\
\hline Ardha shalaba asana & $\sqrt{ }$ & $\sqrt{ }$ & & & $\sqrt{ }$ & \\
\hline Makara asana & $\sqrt{ }$ & $\sqrt{ }$ & $\sqrt{ }$ & $\sqrt{ }$ & & $\sqrt{ }$ \\
\hline \multicolumn{7}{|l|}{ Supine asanas } \\
\hline Pawanamukta series & $\sqrt{ }$ & $\sqrt{ }$ & $\sqrt{ }$ & $\sqrt{ }$ & & $\sqrt{ }$ \\
\hline Pada uttana series & $\sqrt{ }$ & $\sqrt{ }$ & $\sqrt{ }$ & $\sqrt{ }$ & & $\sqrt{ }$ \\
\hline Sethu kriya & & & & & $\sqrt{ }$ & \\
\hline \multicolumn{7}{|l|}{ Relaxation } \\
\hline Savitri pranayama in shava asana & $\sqrt{ }$ & $\sqrt{ }$ & $\sqrt{ }$ & $\sqrt{ }$ & $\sqrt{ }$ & $\sqrt{ }$ \\
\hline Marmanasthanam/kaya kriya & $\sqrt{ }$ & $\sqrt{ }$ & $\sqrt{ }$ & $\sqrt{ }$ & $\sqrt{ }$ & $\sqrt{ }$ \\
\hline \multicolumn{7}{|l|}{ Pranayamas } \\
\hline Mukha bhastrika & $\sqrt{ }$ & & $\sqrt{ }$ & & & $\sqrt{ }$ \\
\hline Chandra nadi/bhedana & $\sqrt{ }$ & $\sqrt{ }$ & & $\sqrt{ }$ & $\sqrt{ }$ & \\
\hline Surya nadi/bhedana & & & $\sqrt{ }$ & & & \\
\hline Pranava & $\sqrt{ }$ & $\sqrt{ }$ & $\sqrt{ }$ & $\sqrt{ }$ & $\sqrt{ }$ & $\sqrt{ }$ \\
\hline Bhramari & $\sqrt{ }$ & $\sqrt{ }$ & $\sqrt{ }$ & $\sqrt{ }$ & $\sqrt{ }$ & $\sqrt{ }$ \\
\hline Nadi shuddhi & $\sqrt{ }$ & $\sqrt{ }$ & $\sqrt{ }$ & $\sqrt{ }$ & $\sqrt{ }$ & $\sqrt{ }$ \\
\hline
\end{tabular}

Table 1: Yoga therapy protocols adopted for different groups with minor individual variations as per their psycho-physical condition and abilities. 
Citation: Bhavanani AB, Ramanathan M, Madanmohan (2013) Immediate Cardiovascular Effects of a Single Yoga Session in Different Conditions. Altern Integ Med 2: 144. doi:10.4172/2327-5162.1000144

Page 3 of 4

subjects had a general schedule of practice. Typical yoga sessions included simple warm ups (jathis and surya namaskar), breath body movement coordination practices (kriyas), static stretching postures (asana), breathing techniques (pranayama), relaxation and chanting. At our centre, we are using the yoga therapy methods and schedules as elucidated in Yoga Chikitsa: The Application of Yoga as a Therapy authored by Yogacharya Dr. Ananda Balayogi Bhavanani, published by Dhivyananda Creations, Pondicherry, India 2013.

Data were assessed for normality using GraphPad InStat version 3.06 for Windows 95, (GraphPad Software, San Diego California USA, www.graphpad.com). As all data passed normality testing by Kolmogorov-Smirnov Test, statistical analysis was carried out using Student's paired t test and 'p' values less than 0.05 were accepted as indicating significant differences for pre-post comparisons (Tables 1 and 2).

\section{Results}

The results are given in Tables 2 and 3 . There were statistically significant $(\mathrm{p}<0.001)$ reductions in all the studied cardiovascular parameters following the yoga session in the overall analysis. The magnitude of reductions differed in the groups, it being more significant in those having hypertension $(n=505)$ and less significant in those having endocrine/skin $(n=230)$ and musculoskeletal $(n=120)$ conditions. It was moderately significant in the normal subjects $(n=582)$ as well as patients having psychiatric $(n=302)$ and respiratory $(\mathrm{n}=157)$ conditions.

\section{Discussion}

There is a healthy reduction in HR, BP and derived cardiovascular indices following a single yoga therapy session. The magnitude of this reduction appears to depend on the pre-existing medical condition as well as the yoga therapy protocol adopted.

Reductions in various cardiovascular parameters following a single session of yoga implies better autonomic regulation of the heart in our subjects. This can be attributed to either an overall increase of parasympathetic tone and/or a reduction in sympathetic tone. RPP and Do $\mathrm{P}$ are indirect indicators of myocardial $\mathrm{O}_{2}$ consumption and load on the heart, thereby signifying a lowering of strain on the heart $[17,18]$. Sympathetic activation is known to increase HR and RPP and decrease overall heart rate variability (HRV). RPP provides a simple measure of HRV in hypertensive patients and is a surrogate marker in situations where HRV analysis is not available [19]. It has also been shown that SDNN and total power of HRV are inversely correlated with mean HR and RPP [17].

\begin{tabular}{|c|c|c|c|c|c|c|}
\hline \multirow[t]{2}{*}{ Groups } & \multicolumn{2}{|c|}{ HR } & \multicolumn{2}{|c|}{ SP } & \multicolumn{2}{|c|}{ DP } \\
\hline & B & A & B & A & B & A \\
\hline $\begin{array}{l}\text { Normal subjects } \\
(n=582)\end{array}$ & $79.93 \pm 8.33$ & $77.14 \pm 7.40^{\star * *}$ & $115.35 \pm 10.42$ & $114.45 \pm 9.36^{*}$ & $74.37 \pm 9.46$ & $73.48 \pm 7.11^{*}$ \\
\hline $\begin{array}{l}\text { Endocrine/skin } \\
(n=230)\end{array}$ & $80.05 \pm 9.46$ & $78.06 \pm 8.54^{* * *}$ & $111.65 \pm 10.64$ & $112.51 \pm 10.04$ & $70.77 \pm 9.00$ & $71.88 \pm 7.40$ * \\
\hline $\begin{array}{l}\text { Respiratory } \\
(n=157)\end{array}$ & $78.55 \pm 7.76$ & $76.36 \pm 6.35^{* *}$ & $112.32 \pm 6.75$ & $111.18 \pm 5.57^{*}$ & $72.94 \pm 6.52$ & $72.79 \pm 5.97$ \\
\hline $\begin{array}{l}\text { Hypertension } \\
(n=505)\end{array}$ & $78.50 \pm 7.38$ & $75.84 \pm 6.71^{* * *}$ & $129.04 \pm 14.65$ & $124.98 \pm 11.35^{* * *}$ & $80.59 \pm 9.96$ & $79.10 \pm 8.39^{* * *}$ \\
\hline $\begin{array}{l}\text { Musculo-skeletal } \\
(n=120)\end{array}$ & $77.78 \pm 7.25$ & $75.80 \pm 6.49$ * & $112.98 \pm 12.14$ & $112.03 \pm 9.13$ & $72.59 \pm 9.12$ & $73.30 \pm 7.31$ \\
\hline $\begin{array}{l}\text { Psychiatry } \\
(n=302)\end{array}$ & $81.53 \pm 9.81$ & $78.66 \pm 9.06$ *** & $115.06 \pm 11.54$ & $112.86 \pm 10.56$ *** & $74.01 \pm 9.18$ & $73.50 \pm 8.02$ \\
\hline $\begin{array}{l}\text { Total } \\
(n=1896)\end{array}$ & $79.55 \pm 8.44$ & $77.00 \pm 7.61^{* * *}$ & $118.13 \pm 13.58$ & $116.39 \pm 11.29$ *** & $75.3 \pm 9.85$ & $74.76 \pm 8.04^{\text {*** }}$ \\
\hline
\end{tabular}

${ }^{*} \mathrm{p}<0.05$

${ }^{* *} p<0.01$

${ }^{* * *} p<0.001$ by Student's paired t test of pre-post comparisons.

Table 2: Heart rate (HR), systolic pressure (SP) and diastolic pressure (DP) before (B) and after (A) a single session of yoga therapy in different groups.

\begin{tabular}{|c|c|c|c|c|c|c|c|c|}
\hline \multirow[t]{2}{*}{ Groups } & \multicolumn{2}{|c|}{ PP } & \multicolumn{2}{|c|}{ MP } & \multicolumn{2}{|c|}{ RPP } & \multicolumn{2}{|c|}{ DoP } \\
\hline & B & A & B & A & B & A & B & A \\
\hline $\begin{array}{l}\text { Normal subjects } \\
(n=582)\end{array}$ & $40.98 \pm 9.70$ & $40.97 \pm 8.03$ & $88.03 \pm 8.66$ & $87.14 \pm 6.97^{\star *}$ & $92.23 \pm 13.11$ & $88.37 \pm 11.84^{\star * *}$ & $70.43 \pm 10.77$ & $67.32 \pm 9.18^{* * *}$ \\
\hline $\begin{array}{l}\text { Endocrine/skin } \\
(n=230)\end{array}$ & $40.88 \pm 7.94$ & $40.63 \pm 8.16$ & $84.39 \pm 8.82$ & $85.42 \pm 7.44$ * & $89.25 \pm 12.98$ & $87.90 \pm 13.04$ & $67.47 \pm 10.22$ & $66.74 \pm 9.77$ \\
\hline $\begin{array}{l}\text { Respiratory } \\
(n=157)\end{array}$ & $39.38 \pm 7.32$ & $38.39 \pm 5.84$ & $86.07 \pm 5.62$ & $85.59 \pm 5.15$ & $88.33 \pm 11.12$ & $84.91 \pm 8.46^{\star *}$ & $67.67 \pm 8.46$ & $65.33 \pm 6.49^{* *}$ \\
\hline $\begin{array}{l}\text { Hypertension } \\
(n=505)\end{array}$ & $48.45 \pm 12.46$ & $45.88 \pm 10.59^{* \star *}$ & $96.74 \pm 10.6$ & $94.39 \pm 8.06^{\star \star *}$ & $101.59 \pm 17.08$ & $94.91 \pm 12.99^{* * *}$ & $76.19 \pm 12.55$ & $71.71 \pm 9.80^{\star \star *}$ \\
\hline $\begin{array}{l}\text { Musculo-skeletal } \\
(n=120)\end{array}$ & $40.39 \pm 9.77$ & $38.73 \pm 6.28^{*}$ & $86.06 \pm 9.13$ & $86.21 \pm 7.39$ & $87.99 \pm 13.34$ & $85.00 \pm 10.76^{*}$ & $67.08 \pm 10.18$ & $65.43 \pm 8.57^{*}$ \\
\hline $\begin{array}{l}\text { Psychiatry } \\
(n=302)\end{array}$ & $41.05 \pm 7.70$ & $39.36 \pm 8.65^{\star *}$ & $87.69 \pm 9.35$ & $86.62 \pm 7.96^{\star *}$ & $93.90 \pm 15.33$ & $88.82 \pm 13.53^{\star * *}$ & $71.55 \pm 11.92$ & $68.16 \pm 10.22^{* * *}$ \\
\hline $\begin{array}{l}\text { Total } \\
(n=1896)\end{array}$ & $42.83 \pm 10.50$ & $41.63 \pm 9.08^{\star \star *}$ & $89.58 \pm 10.8$ & $88.63 \pm 8.20^{* * *}$ & $94.03 \pm 15.28$ & $89.66 \pm 12.76^{\star \star \star}$ & $71.33 \pm 11.64$ & $68.30 \pm 9.63^{* * *}$ \\
\hline
\end{tabular}

${ }^{*} \mathrm{p}<0.05$

${ }^{* *} p<0.01$

${ }^{* * *} p<0.001$ by Student's paired t test of pre-post comparisons.

Table 3: Pulse pressure (PP), mean pressure (MP), rate-pressure product (RPP) and double product (DoP) before (B) and after (A) a single session of yoga therapy in different groups. 
A previous report on the acute effects of one session of hatha yoga practice on blood pressure and other cardiovascular responses in healthy volunteers showed that SP, MP and DP increased significantly during the yoga practice [14]. The elevation in blood pressure due to yoga practice was associated with increases in cardiac output and $\mathrm{HR}$, which are responses similar to those observed in isometric exercise. However it is to be noted that for each posture during the yoga practice, that study continuously measured HR, SP, DP, MP, stroke volume, and cardiac output. On the other hand we have only evaluated the pre-post effects and hence are commenting on the overall effects of the yoga sessions rather than individual practices. The conscious self-effort made in asana practice may be understood as the spanda (tension) component whereas the relaxation of effort (prayatna shaithilya) may be understood as the nishpanda (relaxation) component. Even Maharishi Patanjali tells us that the pair of opposites (dwandwa) is transcended (dwandanabigata) when one perfects the state of asana. Hence, it is essential to physiologically evaluate not only the actual performance of an asana, but also the period of recovery following it.

A study by Telles et al. [20] studied $\mathrm{O}_{2}$ consumption and respiration following four yoga postures interspersed with relaxation and supine relaxation alone, and concluded that the combination of stimulating and relaxing techniques reduced physiological arousal better than the mere practice of relaxation techniques alone [20]. They also pointed out that though the practical performance of yoga techniques seem to be stimulatory in nature, their physiological effects are in fact more relaxatory. This is corroborated by a previous study reporting that shavasana relaxation is enhanced with the addition of savitri pranayama thus decreasing $\mathrm{O}_{2}$ consumption by $26 \%$ [21].

All the yoga therapy protocols adopted at CYTER have a major component of pranayama and yogic relaxation. We hypothesize that this may be the main factor that is producing cardiac autonomic balance in most of our subjects irrespective of their initial condition. We also noticed that the reductions were greater in those who had abnormal readings in the initial testing as opposed to those in whom the initial readings were within normal range. It is traditionally taught that yoga is the state of balance (samatvam yoga uchyate-Bhagavad Gita) and this restoration of physical, mental, emotional and spiritual balance may be the prime factor behind the changes seen across groups.

The above findings of our retrospective data analysis are in agreement with a previous suggestion that yoga appears to modulate stress response systems by reducing perceived stress and anxiety, which in turn, decreases physiological arousal with decreases in HR and BP and respiration [13].

\section{Conclusion}

There is a healthy reduction in HR, BP and derived cardiovascular indices following a single yoga session. The magnitude of this reduction depends on the pre-existing medical condition as well as the yoga therapy protocol adopted. These changes may be attributed to enhanced harmony of cardiac autonomic function as a result of coordinated breath-body work and mind-body relaxation due to yoga.

\section{Acknowledgments}

The authors thank the management and authorities of Sri Balaji Vidyapeeth University for setting up the Centre for Yoga Therapy, Education and Research (CYTER) in Mahatma Gandhi Medical College and Research Institute (MGMCRI). We are grateful to Yogacharini Meenakshi Devi Bhavanani, Director ICYER for her constant motivation and supportive guidance. Thanks are due to Yoga instructors Mrs D Pushpa and Dr. R Balaji for conducting the therapy sessions and for their valuable assistance during recording sessions and data entry.

\section{References}

1. Bhavanani AB (2012) Understanding the science of yoga. Yoga Mimamsa 44 228-245.

2. Khalsa SB (2004) Yoga as a therapeutic intervention: a bibliometric analysis of published research studies. Indian J Physiol Pharmacol 48: 269-285.

3. Vijayalakshmi P, Madanmohan, Bhavanani AB, Patil A, Babu K (2004) Modulation of stress induced by isometric handgrip test in hypertensive patients following yogic relaxation training. Indian J Physiol Pharmacol 48: 59-64.

4. Manjunatha S, Vempati RP, Ghosh D, Bijlani RL (2005) An investigation into the acute and long-term effects of selected yogic postures on fasting and postprandial glycemia and insulinemia in healthy young subjects. Indian $J$ Physiol Pharmacol 49: 319-324.

5. Yang K (2007) A review of yoga programs for four leading risk factors of chronic diseases. Evid Based Complement Alternat Med 4: 487-491.

6. Sengupta P (2012) Health Impacts of Yoga and Pranayama: A State-of-the-Art Review. Int J Prev Med 3: 444-458

7. Madanmohan, Bhavanani AB, Dayanidy G, Sanjay Z, Basavaraddi IV (2012) Effect of yoga therapy on reaction time, biochemical parameters and wellness score of peri and post-menopausal diabetic patients. Int J Yoga 5: 10-15.

8. Streeter CC, Gerbarg PL, Saper RB, Ciraulo DA, Brown RP (2012) Effects of yoga on the autonomic nervous system, gamma-aminobutyric-acid, and allostasis in epilepsy, depression, and post-traumatic stress disorder. Med Hypotheses 78: 571-579.

9. Madanmohan, Bhavanani AB, Zeena S, Vithiyalakshmi L, Dayanidy G (2013) Effects of a comprehensive eight week yoga therapy programme on cardiovascular health in patients of essential hypertension. IJTK 12: 535-554.

10. Innes KE, Bourguignon C, Taylor AG (2005) Risk indices associated with the insulin resistance syndrome, cardiovascular disease, and possible protection with yoga: a systematic review. J Am Board Fam Pract 18: 491-519.

11. Innes KE, Vincent HK (2007) The influence of yoga-based programs on risk profiles in adults with type 2 diabetes mellitus: a systematic review. Evid Based Complement Alternat Med 4: 469-486.

12. Bhavanani AB (2011) Don't put yoga in a small box: the challenges of scientifically studying yoga. Int J Yoga Therap: 21.

13. (2009) Yoga for anxiety and depression. Studies suggest that this practice modulates the stress response. Harv Ment Health Lett 25: 4-5.

14. Miles SC, Chun-Chung C, Hsin-Fu L, Hunter SD, Dhindsa M, et al. (2013) Arterial blood pressure and cardiovascular responses to yoga practice. Altern Ther Health Med 19: 38-45.

15. Huang FJ, Chien DK, Chung UL (2013) Effects of Hatha yoga on stress in middle-aged women. J Nurs Res 21: 59-66.

16. Gothe N, Pontifex MB, Hillman C, McAuley E (2013) The acute effects of yoga on executive function. J Phys Act Health 10: 488-495.

17. Madanmohan, Prakash ES, Bhavanani AB (2005) Correlation between shortterm heart rate variability indices and heart rate, blood pressure indices, pressor reactivity to isometric handgrip in healthy young male subjects. Indian J Physiol Pharmacol 49: 132-138.

18. Bhavanani AB, Sanjay Z, Madanmohan (2011) Immediate effect of sukha pranayama on cardiovascular variables in patients of hypertension. Int J Yoga Therap: 73-76

19. Prakash ES, Madanmohan, Sethuraman KR, Narayan SK (2005) Cardiovascular autonomic regulation in subjects with normal blood pressure, high-norma blood pressure and recent-onset hypertension. Clin Exp Pharmacol Physiol 32 488-494.

20. Telles S, Reddy SK, Nagendra HR (2000) Oxygen consumption and respiration following two yoga relaxation techniques. Appl Psychophysiol Biofeedback 25 221-227.

21. Madanmohan S, Rai UC, Balavittal V, Thombre DP, Gitananda S (1983) Cardiorespiratory changes during savitri pranayama and shavasan. The Yoga Review 3: 25-34 